\title{
A novel on Newton-Rapson iterative interpolation algorithm for NURBS curve
}

\author{
Wan-Jun Zhang ${ }^{1,2,3, a}$, Dong-mei $X^{1, b}$,Xin-hong Meng ${ }^{1, c}$ \&Feng Zhang ${ }^{2, d}$ \\ 1 Quanzhou Institute of Information Engineering, \\ 2 Lanzhou Industry and Equipment Co. ,Ltd. , Lanzhou 730050, China, \\ 3 School of Mechanical Engineering, Xian Jiao tong University, China \\ agszwj_40@163.com, ${ }^{b} x d m 33 @ 163 . c o m,{ }^{c}$ meng2008xinhong@126.com, ${ }^{d} 116543048 @ q q . c o m$
}

Keywords: NURBS curve,Newton-Rapson iterative, interpolation algorithm .

\begin{abstract}
Linear or cicular interpolator are undersirable for high-speed ,precision and advanced CNC machining tools of $2 \mathrm{D}$ or $3 \mathrm{D}$ surfaces for the following resons: interpolation time bigger, calculation more complicated, and curve chord are changed. In the papers ,the Newton-Rapson iterative interpolation algorithm for NURBS curve is introduced. We can use Newton-Rapson iterative that calculate $\left(x_{i}, y_{i}, z_{i}\right)$. Simulation results show that the proposed NURBS curve interpolator meet the high-speed and high-accuracy interpolation requirements of CNC systems. The interpolation of NURBS curve should be finished. The simulation results show that the algorithm is correct; it is consistent with a NURBS curve interpolation requirements.
\end{abstract}

\section{Introduction}

Modern CNC manufacturing systems can provide linear interpolations and circular interpolations for high-speed ,precision and advanced CNC machining tools of 2D or 3D surfaces for the following resons [1-3]: interpolation time bigger, calculation more complicated, and curve chord are changed. NURBS have been used by many CAD/CAM systems as a 3D geometry reprentation ,which became CNC machining standard. Researchers[4-6] have researched interpolation algorithms for NURBS curves. Here has been attempts to change the feed rate in NURBS interpolation [7].Literature [8-9] were given some NURBS interpolation algorithms, which makes NC programming complicated. Shpitalni et al. [9] derived the same interpolation algorithm by using Taylor's expansion. Houng and Yang [10] were given Cubic spline curve interpolator by using Euler algorithm. Lo and Chung[11] proposed the error interpolation algorithm which error calculations changed by curve chord.

On the basis of the research above, a Newton-Rapson iterative interpolation algorithm for NURBS is presented in this novel. The simulation results show that the algorithm is consistent with a NURBS curve interpolation requirements. This interpolation algorithm cannot meet the highspeed and high-accuracy NURBS curves interpolation requirements.

\section{NURBS Interpolator}

In this note, NURBS curve is used to represent a parametric adaptive of curve, and it is introduced first. Supposed $p(u)$ can be represented NURBS curve. While NURBS [3] are parametrically mathematical definition by the following Eq.(1):

$$
p(u)=\sum_{i=0}^{n} \omega_{i} d_{i, k}(u) / \sum_{i=0}^{n} \omega_{i} N_{i, k}(u)=\sum_{i=0}^{n} p_{i} N_{i, k}(u)
$$

Where $u$ is cubic time NURBS curve each parameter, $\mathrm{k}$ the order of NURBS curve $p_{i}$ is the control points, $\omega_{i}$ is the weight vector , $N_{i, k}(u)$ is the blending function .

In three dimension, NURBS curve is described as 


$$
P(u)=x(u) i+y(u) j+z(u) k=[x(u), y(u), z(u)]^{T}
$$

Since NURBS curve feed speed $V\left(\boldsymbol{u}_{i}\right)$ can be represented as

$$
V\left(\boldsymbol{u}_{i}\right)=\left|\frac{d P(u)}{d t}\right|=\left|\frac{d P(u)}{d u}\right| \frac{d u}{d t}=\left(\frac{d X_{i}}{d u} i+\frac{d y_{i}}{d u} j+\frac{d Z_{i}}{d u} k\right) \frac{d u}{d t}
$$

Where $\|\cdot\|$ denotes vector modulo and therefore

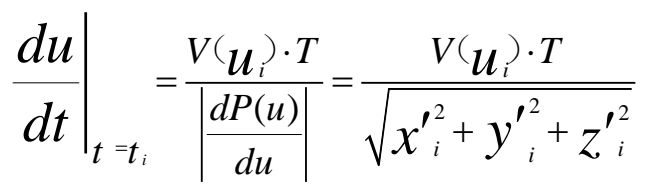

According to the parameter $u_{i}$ at $t=t_{i}$ and the first-order, second-order derivate of function $u_{i+1}$ with $t$, so, we can also calculate $u_{i+1}$ at $t=t_{i+1}$ by Taylor's expansion as equation(5)

$$
u_{i+1}=u_{i}+\left.\frac{d u}{d t}\right|_{t=t_{i}} T+\left.\frac{1}{2} \frac{d^{2} u}{d t^{2}}\right|_{t=t_{i}} T^{2}+\text { H.O.T. }
$$

H.O.T. denotes the higher order terms. Second-order derivate of $u$ is given as [3]

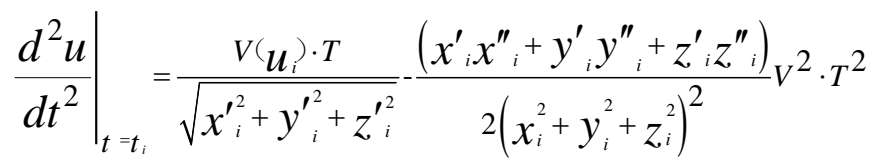

Also, the first-order interpolation equation for NURBS curve is obtained by substituting Eq.(5)into Eq.(6) can be represented as follows:

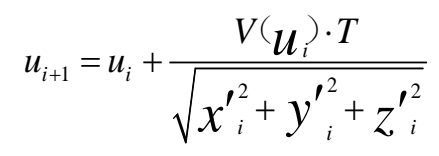

We can get second -order interpolation equation for NURBS curve.

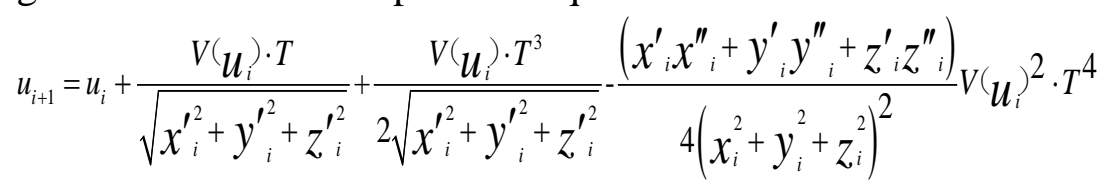

By using Eq.(8) and Eq.(9) ,the common parameter $\left(x_{i}, y_{i}, z_{i}\right)$ is updated each sampling interpolation time $T$ to cope with feed speed $V(u)$. Therefore the NURBS curve $\left(x_{i}, y_{i}, z_{i}\right)$ to reach can be calculated using Eq.(2).In the research, the NURBS curve position $\left(x_{i}, y_{i}, z_{i}\right)$ can be calculated by using Newton-Rapson iterative interpolation method. Where $V(u)$ can be represented feed speed command in machining process.

\section{Newton-Rapson iterative interpolation algorithm}

\section{Newton-Rapson iterative interpolation method.}

In this mathematical model of Newton-Rapson iterative interpolation algorithm, as shown in Fig.1.If we can suppose NURBS curve with existing maximum deceleration piont $A_{1}\left(u_{i}\right)$ and minimum acceleration piont $A_{2}\left(u_{i+1}\right)$, the length of piont $A_{1}\left(u_{i}\right)$ and piont $A_{2}\left(u_{i+1}\right)$ should equal to $A=\left\|A_{1}\left(u_{i}\right)\right\|-\left\|A_{2}\left(u_{i+1}\right)\right\|=\left\|P(u)_{i}\right\|$. 


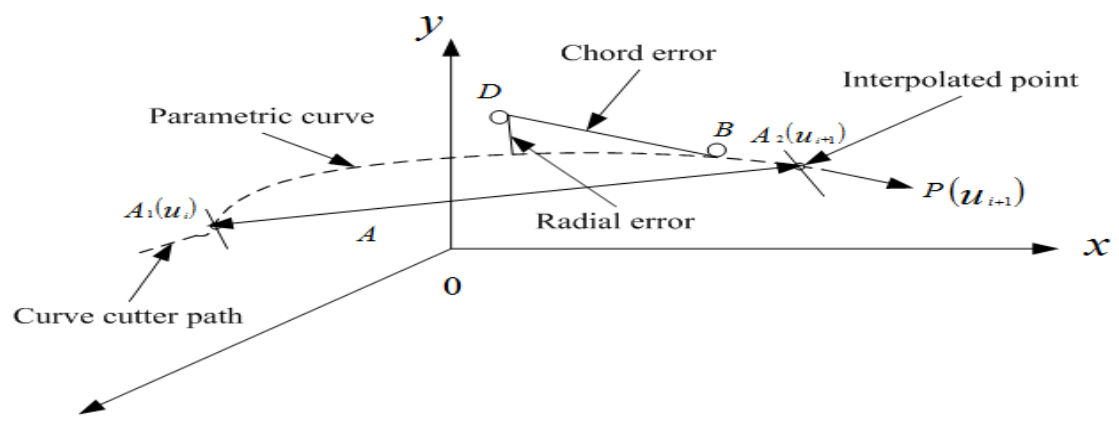

Fig. 1 The mathematical model of NURBS curve interpolation

where, $A=\left\|A_{1}\left(u_{i}\right)\right\|-\left\|A_{2}\left(u_{i+1}\right)\right\|=\int_{u_{i}}^{u_{i+1}}\left\|\frac{d p}{d u}\right\| d u$. when $A-\int_{u_{i}}^{u_{i+1}}\left\|\frac{d p}{d u}\right\| d u=0$, that is $\left\|A_{1}\left(u_{i}\right)\right\|=\left\|A_{2}\left(u_{i+1}\right)\right\|, \quad u$ can be solved, $\left(x_{i}, y_{i} z_{i}\right)$ can obtain the value.

Set Newton-Rapson iterative $f(u)=A-\int_{u_{i}}^{u_{i+1}}\left\|\frac{d p}{d u}\right\| d u, u_{i+1}^{-} u_{i}=\frac{f\left(u_{i}\right)}{f^{\prime}\left(u_{i}\right)}, \quad i \geq 1$.

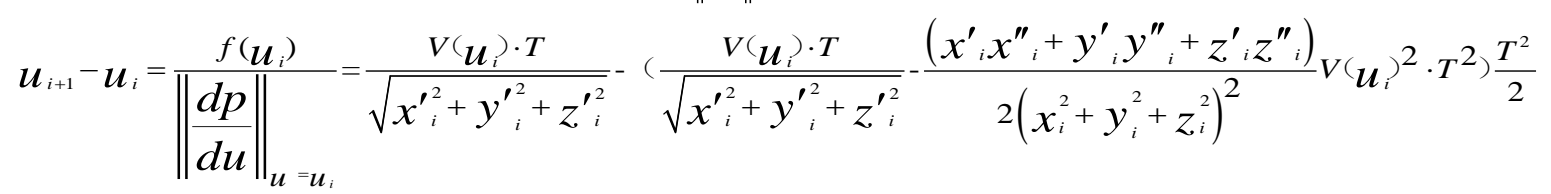

Newton-Rapson iterative exist a $\varepsilon, \frac{\left|u_{i+1}-u_{i}\right|}{u_{i}}<\varepsilon, \quad u_{i} \neq 0$. So,

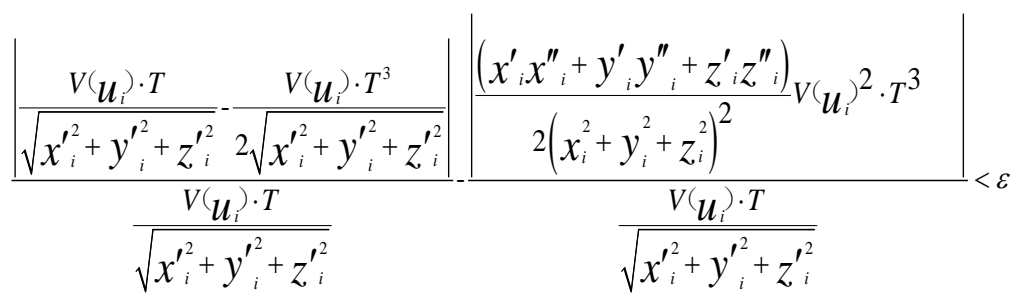

As shown in Eq.(8)and(9),we can get Eq.(11), Eq.(10) could calculated $u_{i}$, we should know $\left(X_{i}, y_{i}, z_{i}\right)$.

\section{Newton-Rapson iterative interpolation algorithm for chor error.}

If NURBS curve interpolation can finished, the feed rate should keep costant as $V(u)$ during interpolation process. In Fig. $1, \rho_{i}$ is radius of curvature at $u_{i}, P\left(u_{i}\right)$ and $P\left(u_{i+1}\right)$ are the NURBS curve interpolated.And $\delta_{i}$ is derived as

$$
\delta_{i}=\rho_{i}-\sqrt{\rho_{i}^{2}-\left(\frac{V(u) T}{2}\right)^{2}}
$$

The feed speed $V(u)$ corresponding to the chord $\delta_{i}$ is derived as

$$
\text { If } \delta_{i}<\delta_{\max } \text {, where } V_{\max }\left(u_{i}\right)=\frac{2}{T} \sqrt{\rho^{2}-\left(\rho_{i}-\delta_{\max }\right)^{2}}=\frac{2}{T} \sqrt{\delta_{\max }\left(2 \rho_{i}-\delta_{\max }\right)}
$$

Eq.(13) expresses that NURBS curve speed rate $V\left(u_{i}\right)$ should be included adaptively accrding to $\rho_{i}$ and $\delta_{i}$. So, $\delta_{i}$ can be represented the tolerance of the chord error. where $F$ is the feed speed command. Since NURBS curve feed speed $V(u)$ is changed according to the radius of curvature, the following feed speed rule is proposed for parametric curves:

$$
V\left(u_{i}\right)= \begin{cases}F & \text { if } \delta_{i} \leq \delta_{\max } \\ \frac{2}{T} \sqrt{\rho^{2}-\left(\rho_{i}-\delta_{\max }\right)^{2}} & \text { otherwise }\end{cases}
$$




\section{Interpolation algorithm flow chart}

Figure 3 for Flowchart of algorithm. Newton-Rapson iterative interpolation method is explained as follow:

Step1: the input date are from NURBS curve parameters model such as NURBS curve control points, weight vector and so on.

Step2: the NURBS curve position $\left(X_{i}, y_{i}, z_{i}\right)$ can be calculated by using Newton-Rapson iterative interpolation method which exist a $\varepsilon$.

Step3: According to Eq.(14), the max feed speed and the min feed speed that we can be known ,NURBS curve interpolation is finished.

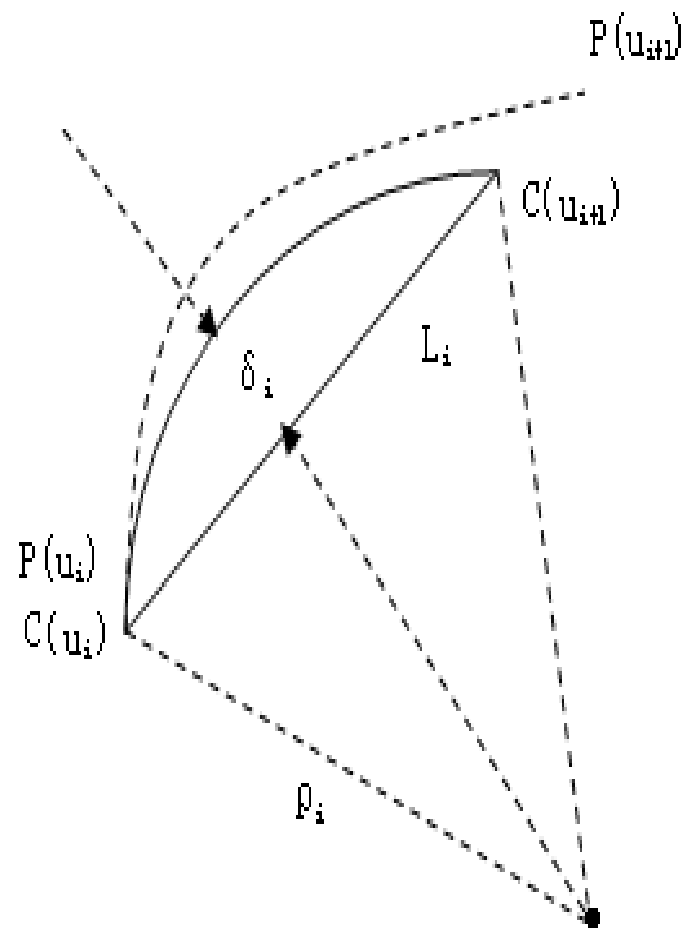

Fig.2 NURBS curve chord $\delta_{i}$

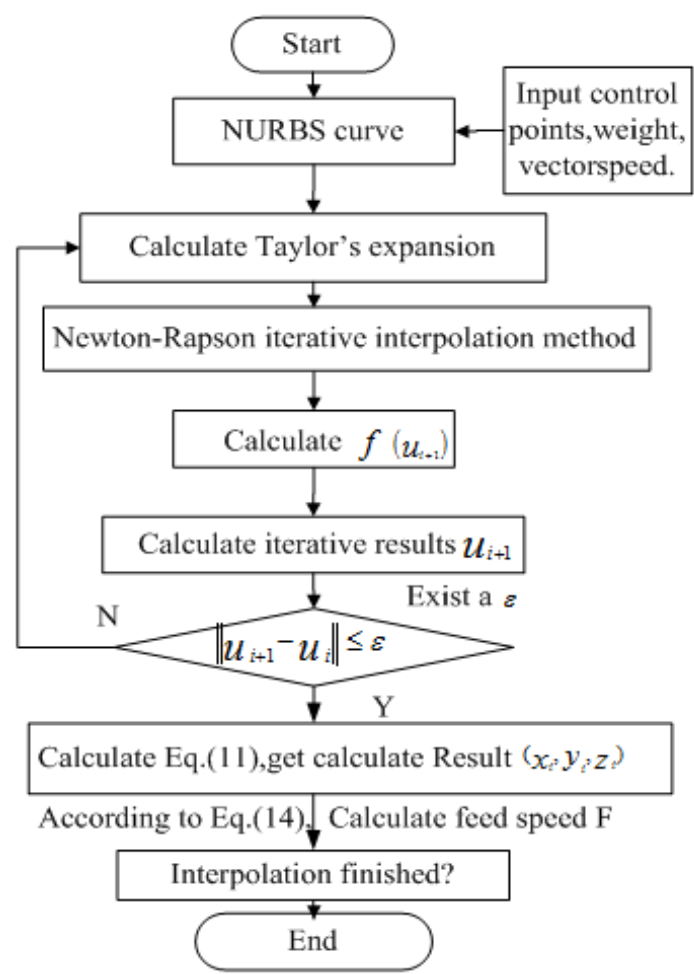

Fig .3 Flowchart of algorithm

\section{Experiment simulation and data analysis}

In this simulation ,this interpolation scheme is realized on the motion controller developed by our own lab, based on DSP TMS20C543,Development environment is a PC with AMD Sempron $2,800+2.1 \mathrm{Ghz}$ CPU,2GB RAM, and main frequency is $1.44 \mathrm{MHz}$,machine tool is machine center. Machining parameters and dynamics parameters are shown in Table 1.

In the paper, the interpolation of a Newton-Rapson iterative interpolation curve is utilized as an example to the Newton-Rapson iterative algorithm. The control points ,weight vector, and knot vector of NURBS for the provided example are assigned as follows: The control pointsare $p_{0}(0,0,0), p_{1}(1,1,2), p_{2}(2,1,2), p_{3}(3,1.5,2), p_{4}(3,2,2.5), p_{5}(3.5,3,3)$ (unit: $\left.\mathrm{mm}\right)$, The weight vector is

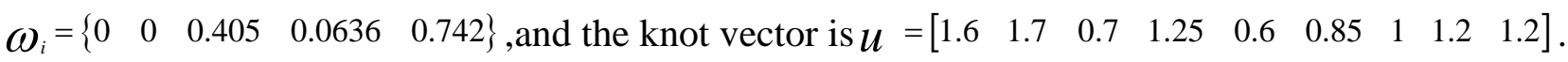

Tab. 1 Machining parameters and dynamics parameters

\begin{tabular}{|c|c|c|c|c|c|c|}
\hline$J\left(\mathrm{~mm} \cdot \mathrm{s}^{-2}\right)$ & $F\left(\mathrm{~mm} \cdot \mathrm{s}^{-1}\right)$ & $T$ (ms) & $\delta_{\max }(\mu \mathrm{m})$ & $V_{x \max }\left(\mathrm{mm} \cdot \mathrm{s}^{-1}\right)$ & $V_{y \max }\left(\mathrm{mm} \cdot \mathrm{s}^{-1}\right)$ & $V_{z \max }\left(\mathrm{mm} \cdot \mathrm{s}^{-1}\right)$ \\
\hline 48000 & 200 & 1 & 1 & 60 & 80 & 50 \\
\hline
\end{tabular}


feed rate vale of y-axis, $V_{z \max }$ the max feed rate vale of z-axis. By Using computer soft in NURBS curve interpolation, as shown in Tab.2, Figure 4, Figure 5andFigure 6.

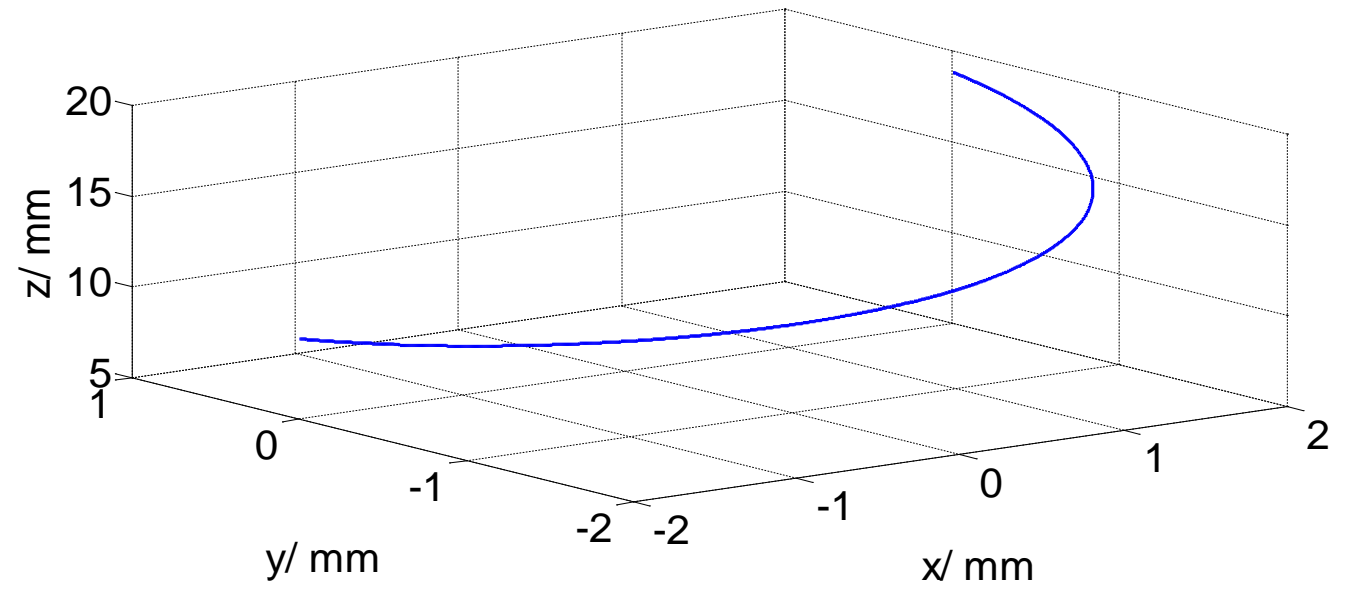

Fig. 4 NURBS curve interpolation

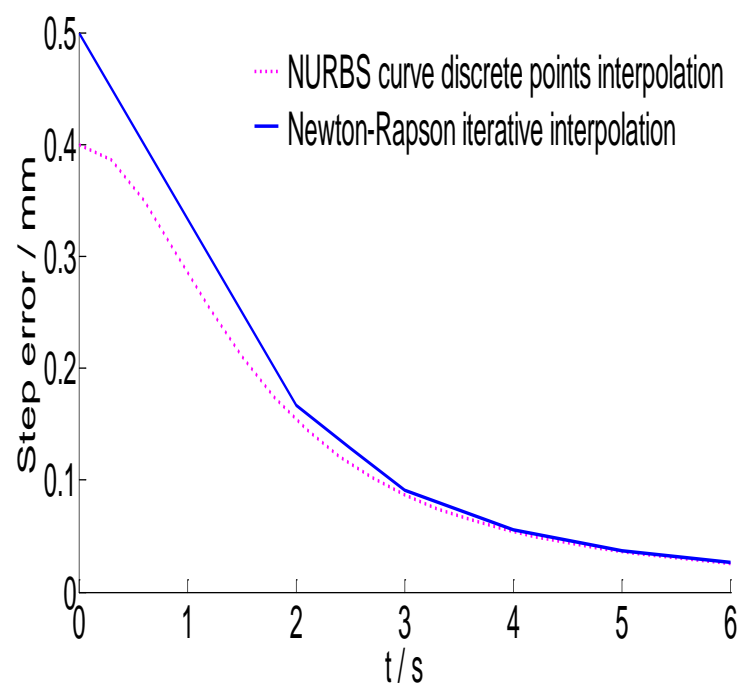

Fig. 5 Step error (mm)

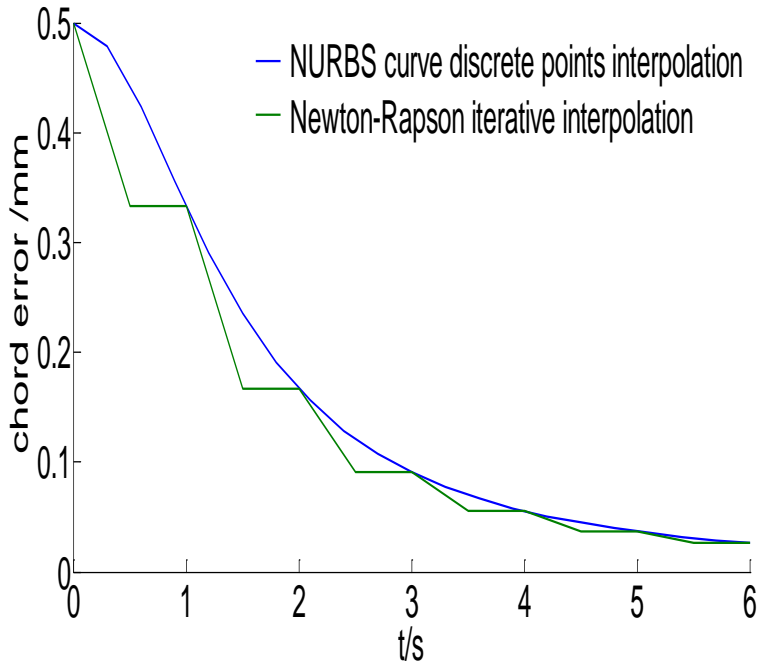

Fig. 6 Chord error(mm)

Tab.2 Table analysis of interpolation of NURBS curve results

\begin{tabular}{|c|c|c|c|c|}
\hline Parameters algorithms & $\begin{array}{c}\text { Interpolation } \\
\text { time(s) }\end{array}$ & $\begin{array}{l}\text { Step error } \\
(\mathrm{mm})\end{array}$ & $\begin{array}{l}\text { Max chord } \\
\text { error }(\mathrm{mm})\end{array}$ & $\begin{array}{l}\text { Max contour } \\
\text { error }(\mathrm{mm})\end{array}$ \\
\hline $\begin{array}{l}\text { NURBS curve discrete } \\
\text { points interpolation }\end{array}$ & 17.84 & 0.05521 & 9.0801 & 0.712 \\
\hline $\begin{array}{l}\text { Newton-Rapson } \\
\text { iterative interpolation }\end{array}$ & 15.5 & 0.0125 & 7.9801 & 0.46 \\
\hline
\end{tabular}

As can be seen from Fig. 4, Fig. 5, Fig.6 and table 2, in the process of the interpolation, interpolation time reduced, max chord error and max contour error deceased, the special compensation allowed values in the interpolation process significantly reduced as the value of $0.0125 \mathrm{~mm}$, chord error max value is 7.980 , which meet the expected to interpolation, i.e. to reduce the compensation error and interpolation step chord error. To verify the high efficiency and reliability of this Newton-Rapson iterative interpolation algorithms are applied in the experiments to make a comparison .It can be seen that Newton-Rapson iterative interpolation algorithm is feasible and efficent.

\section{Summary}

In the paper, a novel on Newton-Rapson iterative interpolation algorithm for NURBS curve is introduced. The common parameter $\left(X_{i}, y_{i}, z_{i}\right)$ is updated each sampling interpolation time $T$ to 
cope with feed speed $V(u)$.Starting from the definition of the interpolation parameter of NURBS curves, the NURBS curve position $\left(X_{i}, y_{i}, z_{i}\right)$ can be calculated by using Newton-Rapson iterative interpolation method which exist a $\varepsilon$. Where $V(u)$ can be represented feed speed command in machining process. Simulation results show that the proposed NURBS curve interpolator meet the high-speed and high-accuracy interpolation requirements of CNC systems. In addition ,NC machining time can be reduced. Implementation on NC machine has proven the feasibility of a developed interpolation algorithm.

\section{Acknowledgements}

The authors thank the financial supports from National Natural Science Foundation of China(Grant no. 51165024) and Science and Technology Major Project of "High-grade NC Machine Tools and Basic Manufacturing Equipment” (2010ZX040001-181).

\section{References}

[1] Fang Yi-Xiang, Liu Wen-Xue. Based on the geometric properties of the cubic uniform B-Spline curve structure description[J]. Journal of engineering graphics, 2 , pp.96-102, 2006.

[2] Zhang Wan-Jun, Hu Chi-Bing, Zhang Feng , et al . Honing machine motion control card three B spline curve method of interpolation arithmetic for CNC system [J]. Chinese Journal of Manufacturing Technology \& Machine Tool , 8(8), pp.40-43,August 2012.

[3] Zhang Wan-Jun, HU Chi-bing, WU Zai-xin, et,al. Research on modification algorithm of Three B Spline curve interpolation technology [J]. Chinese Journal of Manufacturing Technology \& Machine Tool , 2 pp.141-143,Feburary 2013.

[4] Zhang Wanjun, Zhang Feng , Zhang Guohua. Research on a algorithm of adaptive interpolation for NURBS curve. [J].Applied Mechanics and Materials , Vol. 687-691, pp.1600-1603, December 2014.

[5] Zhang Wan-Jun, Zhang Feng, Zhang Guohua. Research on modification algorithm of Cubic Bspline curve interpolation technology. [J].Applied Mechanics and Materials, Vol. 687-691, pp.15961599, December 2014.

[6] Kong Fan-Guo, Hao Shang-Hua, ZhongYan-Zhi. NURBS curve interpolation algorithm to achieve the VC [J]. China new technology and new products, 17, pp.8-9, 2009.

[7] Ye Bo-Sheng,Yang Shu-Zi. CNC system in cubic B-Spline curve interpolation method [J ]. China Mechanical Engineering, 9 ( 3) , pp. 42 - 43, 1998.

[8] Li He-Cheng, Wang Yu-Ping, An interpolation based genetic algorithm for sloving nonlinear bilevel programming problems.Chinese Journal of Computers, 31(6), pp.910-918, June 2008.

[9] Shpitalni M, Koren Y, Lo CC. Realtime curve interpolators. Computer- Aided Design, 26, pp.832-838, 1994.

[10] Huang JT, Yang DCH. A generalized interpolator for command generation of parametric curves in computer controlled machines. Japan/USA Symposium on Flexible Automation,1(1), pp.393-399, January1992.

[11] Lo CC, Chung CY. Curve generation and control for biaxial machine tools. J. CSME , 18, pp.175-182, 1997.

[12] Zhang Wan-Jun, Zhang Feng, Zhang Wan-Liang. Research on a NURBS curve of timing / interrupt interpolation algorithm for CNC system [J]. Chinese Journal of Manufacturing Technology \& Machine Tool , 4(4), pp.40-43,April 2015. 